\title{
Alternative Approaches to High Energy Density Fusion
}

J. H. Hammer

November 18, 2013

The 8th International Conference on Inertial Fusion Sciences and Applications (IFSA)

Nara, Japan

September 8, 2013 through September 13, 2013 
This document was prepared as an account of work sponsored by an agency of the United States government. Neither the United States government nor Lawrence Livermore National Security, LLC, nor any of their employees makes any warranty, expressed or implied, or assumes any legal liability or responsibility for the accuracy, completeness, or usefulness of any information, apparatus, product, or process disclosed, or represents that its use would not infringe privately owned rights. Reference herein to any specific commercial product, process, or service by trade name, trademark, manufacturer, or otherwise does not necessarily constitute or imply its endorsement, recommendation, or favoring by the United States government or Lawrence Livermore National Security, LLC. The views and opinions of authors expressed herein do not necessarily state or reflect those of the United States government or Lawrence Livermore National Security, LLC, and shall not be used for advertising or product endorsement purposes. 


\title{
Alternative Approaches to High Energy Density Fusion
}

\author{
J Hammer \\ Lawrence Livermore National Laboratory, Livermore California 94550, USA
}

E-mail: hammer2@1ln1.gov

\begin{abstract}
This paper explores selected approaches to High Energy Density (HED) fusion, beginning with discussion of ignition requirements at the National Ignition Facility (NIF). The needed improvements to achieve ignition are closely tied to the ability to concentrate energy in the implosion, manifested in the stagnation pressure, $P_{\text {stag }}$. The energy that must be assembled in the imploded state to ignite varies roughly as $P_{\text {stag }}{ }^{-2}$, so among other requirements, there is a premium on reaching higher $P_{\text {stag }}$ to achieve ignition with the available laser energy. The U.S. inertial confinement fusion program (ICF) is pursuing higher $P_{\text {stag }}$ on NIF through improvements to capsule stability and symmetry. One can argue that recent experiments place an approximate upper bound on the ultimate ignition energy requirement. Scaling the implosions in spatial, temporal and energy scales shows that implosions of the demonstrated quality ignite robustly at 9-15 times the current energy of NIF. While lasers are unlikely to reach that bounding energy, it appears that pulsed power sources could plausibly do so, giving a range of paths forward for ICF depending on success in improving energy concentration. In this paper, I show the scaling arguments then discuss topics from my own involvement in HED fusion. The recent Viewfactor experiments at NIF have shed light on both the observed capsule drive deficit and errors in the detailed modelling of hohlraums. The latter could be an important factor in the inability to achieve the needed symmetry and energy concentration. The paper then recounts earlier work in Fast Ignition and the uses of pulsed power for HED and fusion applications. It concludes with a description of a method for improving pulsed power driven hohlraums that could potentially provide a factor of 10 in energy at NIF-like drive conditions and reach the energy bound for indirect drive ICF.
\end{abstract}

\section{Introduction}

High Energy Density (HED) fusion research is in an exciting time given the progress on the National Ignition Facility (NIF). Ignition remains elusive, yet the important milestone of alpha particle heating - doubling the yield due to self-heating - appears likely to be within reach. The basic requirements for ICF ignition are well known [1]. The temperature of the ignition region must be high enough to exceed radiative losses and give high fusion reactivity, e.g., a burn averaged value of near $4.5 \mathrm{keV}$ or higher. Most of the alpha particle energy must be deposited in the ignition spot and the Lawson parameter, the product of pressure and energy confinement time, $P \tau$, must be high enough to give significant gain. The latter two conditions can usually be expressed in terms of $\rho R$, or density times radius in $\mathrm{g} / \mathrm{cm}^{2}$, with $\rho R \sim 0.3$ in the hot spot to trap alphas and the total $\rho R>1$ to give reasonable gain. 
If we focus on the energy requirements of the hot spot, then a simple relationship between energy and the stagnation pressure of the capsule exists. Since $P_{\text {stag }} \propto \rho T$ for high temperature matter, and the hot spot energy is proportional to the product of pressure and volume, we have the result:

$$
E_{\text {Hotspot }} \propto \rho T R^{3}=\frac{(\rho R)^{3} T}{\rho^{2}} \propto \frac{(\rho R T)^{3}}{P_{\text {Stag }}{ }^{2}} \propto P_{\text {Stag }}{ }^{-2}
$$

The last relationship in equation (1) follows if we take $\rho R$ and $T$ to be determined by the ignition requirements. For instance at $\rho R=0.3, T=4.5 \mathrm{keV}$ we have $E_{\text {Hotspot }} \cong 70 \mathrm{~kJ} * P_{\text {Stag }}{ }^{-2}$ for $P_{\text {stag }}$ in units of $100 \mathrm{GBar}$. The total energy invested in fuel internal energy is larger than $E_{\text {Hotspot }}$ because of the surrounding dense, cold fuel layer needed for inertial confinement. High gain hot-spot targets could be dominated by the energy of the cold fuel, but ignition targets at the NIF have cold fuel energy comparable to hot spot energy. Fast ignition designs have similar scaling but require higher $\rho R$ in the ignition spot.

Equation (1) shows the importance of reaching high stagnation pressures to enable ignition at reasonable energy. At $400 \mathrm{~GB}$, typical of ignition designs, the hot spot energy required is about 4.4 $\mathrm{kJ}$, which should be achievable at NIF. Most experiments to date, on the other hand, have been limited to $P_{\text {stag }} \lesssim 150 G B$ [2]. Many factors such as implosion symmetry, hydrodynamic instability, shock mis-timing or fuel pre-heat could contribute to low $P_{\text {stag }}$, and improved understanding of these effects are among the main thrusts of the experimental program moving forward. One simple way of looking at the issue is through energy conservation. The stagnated state energy should approximately equal the $P d V$ work done by the implosion:

$$
P_{\text {Stag }} V_{\text {Stag }} \propto P_{\text {Drive }} V_{0} \rightarrow P_{\text {Stag }} \propto P_{\text {Drive }}\left(V_{0} / V_{\text {Stag }}\right) \rightarrow P_{\text {Stag }} \propto P_{\text {Drive }} C^{3}
$$

where $P_{\text {Drive }}$ is the average drive pressure, $\sim T_{R}^{3.5}$ for indirect drive. $V_{0}$ and $V_{\text {Stag }}$ are the initial and stagnation volumes and $C$ is the fuel convergence ratio. Note that $C$ defined this way differs from the usual hot spot convergence ratio. One caveat with this expression is that many NIF implosions have missing energy, i.e., kinetic energy present in the implosion is incompletely converted to internal energy [2]. The drive temperature and pressure are limited by available power or laser intensity limits within the hohlraum, so increasing $P_{\text {stag }}$ depends critically on reducing sources of asymmetry, instability, etc. that limit $C$ or disrupt the efficient energy conversion assumed in equation (2).

The High Foot experiments [3] recently conducted at NIF, so named for the higher drive temperature and shock pressure during the foot of the pulse, are interesting not only in being the highest yield to date, but because they agree well with simulations for most measured and inferred quantities such as yield, $\rho R$, burn temperature, and pressure. They thus represent validation of hydrodynamic and burn models for their operating regime of implosions on the NIF. With a burnaveraged $P_{\text {stag }}$ of $100-150 \mathrm{~GB}$, and $C \sim 17$ they are below ignition requirements at the available energy, but arguably they set a bound on what energy would be needed to ignite at the values of $C$ and $P_{\text {stag }}$ that have already been demonstrated. Equation (1) suggests that bound is about one order of magnitude larger than the existing NIF implosions, however we can use simulations to better quantify the issue. Starting with a 1 dimensional radiation hydrodynamics model of the High Foot implosion experiment N130710, we can scale the implosion while preserving drive pressure, shock-timing, $C$ and $P_{\text {stag }} \cong 119 G B$ using Euler scaling:

$$
R \rightarrow S R, \quad t \rightarrow S t, \quad P \rightarrow P, \quad E \rightarrow S^{3} E
$$

where $S$ is the spatial/temporal scale factor, giving scaling as $S^{3}$ in energy. The calculations do not include the ablator, but use a pressure drive extracted at the fuel-ablator interface since radiationdriven ablation does not strictly follow Euler scaling. Heat conduction in the hot spot also does not follow Euler scaling, but has little effect on $C$ and $P_{\text {stag }}$. Burn-off calculations confirm that $C$ and $P_{\text {stag }}$ are preserved for $S<3$. The results of the scaling are shown in figure 1, confirming the expectation from equation (1). Interestingly, the bounding energy for ignition (and high yield) from this argument may be within the capabilities of future laboratory drivers. The dashed lines in figure 1 
show the drive energy from a hypothetical $16 \mathrm{MJ}$ pulsed power source discussed below. As indicated in figure 2, the path toward ignition may come through either raising the drive energy, improving implosion quality or both. In that vein, the recent experiment N130927 achieved preliminary values of inferred pressure of $\sim 150 \mathrm{~GB}$, suggesting a revised bounding energy a $\sim 40 \%$ lower than shown in figure 1.
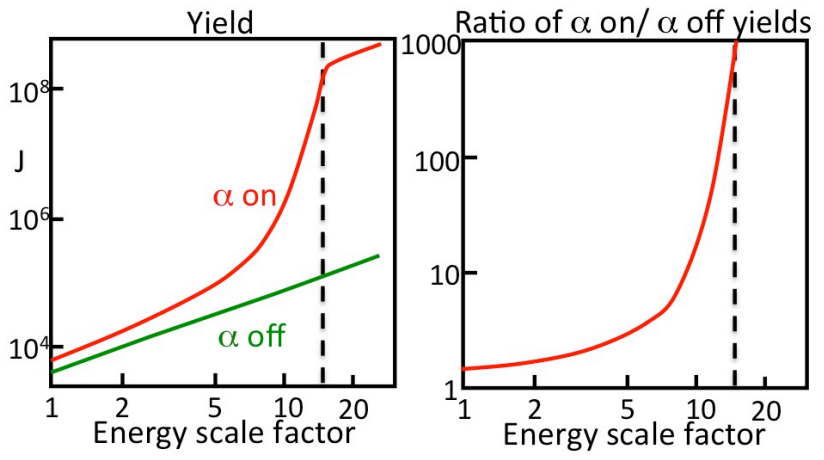

Figure 1. Yields with alpha deposition on/off and the ratio of alpha-on to alpha-off yield from hydrodynamically scaled High Foot capsule, versus the energy scale factor, $S^{3}$

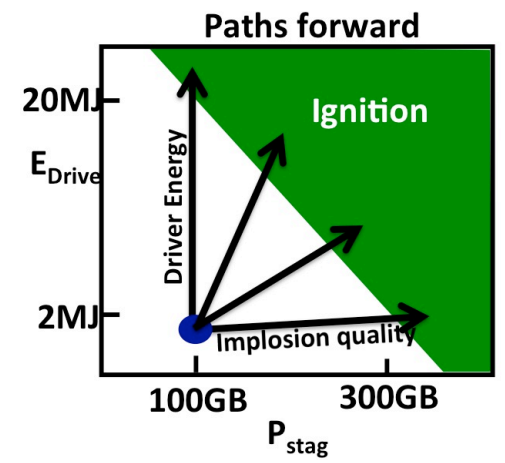

Figure 2. Possible paths toward ignition

\section{Viewfactor Experiments}

A viewfactor experiment, shown in Figure 3, employs an ignition-scale cryogenic hohlraum with one end cut off, exposing the interior and far laser entrance hole (LEH) to views that allow absolute measures of x-ray flux with Dante diagnostics as well as x-ray imaging. The purpose is to characterize the $\mathrm{x}$-ray drive from the viewpoint of a capsule, as opposed to the usual measurement of the drive through the LEH from which the capsule drive is inferred. The experiments are designed to create a plasma and radiation environment as close as possible to the ignition target while allowing for the improved view of the hohlraum interior. The experiments are motivated by inconsistencies between the LEH-inferred drive and the implosion trajectories of capsules. As reported in greater detail in by M Schneider at another paper at this conference, viewfactor experiments are indeed largely consistent with a lower capsule drive than the LEH-inferred drive. The drive deficit of 15-25\% represents much, although perhaps not all, of the discrepancy between simulations and the observed implosion trajectory. X-ray imaging diagnostics provide a wealth of data about the uniformity and spatial location of hohlraum reemission, as well as clear signatures of the effect of cross beam energy transfer on beam spot emission.

Figure 4 shows the ratio of measured to calculated drive through the open end of the hohlraum, indicating a discrepancy largely consistent with drive multipliers used to match observed capsule trajectories. In contrast, the ratio of measured to calculated drive through the LEH end is close to 1, as usually found for cryogenic hohlraums. X-ray imaging suggests the discrepancy is due to cancelling errors from over-calculating both LEH closure and the internal hohlraum drive. As shown in Figure 3, $\mathrm{x}$-ray images at energies near the peak of the wall reemission spectrum show structure which can imprint asymmetries on the capsule. Variation in wall reemission is expected as shown in the bottom image in Figure 3, but the detailed pattern differs significantly from the calculation. Images at higher x-ray energies show that the extent and location of the "gold bubble" produced by the laser spots also differ from calculations. The pattern of x-ray emission near the wall determines the time dependent implosion symmetry: if the emission is decomposed into spherical harmonics, the non-uniformities in intensity distribution at the capsule are smoothed by factors of a few at low mode number, $l$ to an order of magnitude or more at high $l$. The resulting net drive flux asymmetry is inversely proportional to the 
achievable convergence, $C$, so improvements in hohlraum modeling and control appear to be critical to increasing $P_{\text {stag }}$, as indicated by equation 2 , and the chances of ignition suggested by equation 1 .

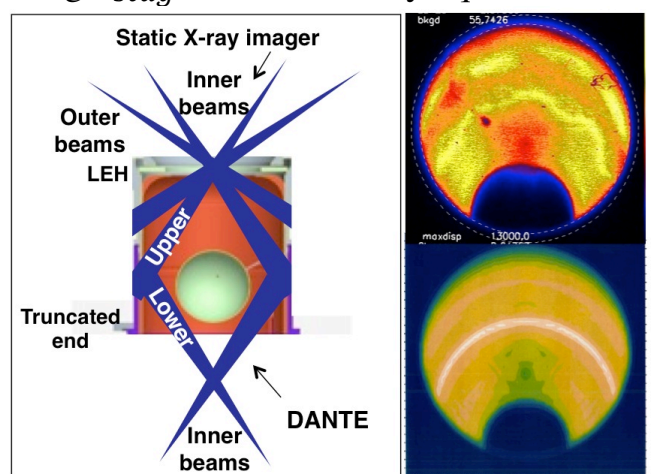

Figure 3. Viewfactor target and time integrated images through the open end at $870 \mathrm{eV}$ for shot N120816, experiment(top) and 2D simulation(bottom). Lineouts show $20 \%$ brightness variation, differing from simulated wall reemission, especially for inner beams.

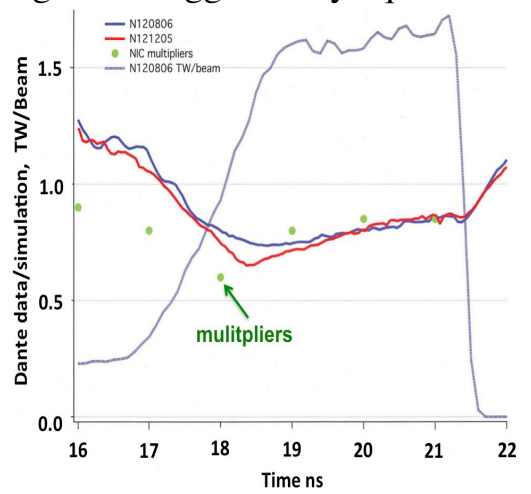

Figure 4. The ratio of measured to calculated open end $\mathrm{x}$-ray flux for viewfactor experiments N120806 and N121205, along with drive multipliers inferred from capsule trajectories. The laser pulse is shown for reference.

\section{Fast Ignition}

Consider another chapter in the fusion story: fast ignition. Around 1990, two technological developments of interest were taking place. First, the phenomenon of radiative collapse in a cryogenic deuterium or deuterium-tritium fiber z-pinch was being studied [4], and second, the new chirped-pulse laser technology had been developed. The latter was projected to enable delivery of $10 \mathrm{~kJ}$ or more in a few picoseconds with a spot size of order $10 \mu \mathrm{m}$.

One dimensional radiation magnetohydrodynamic (MHD) models suggested that it might be possible to compress a cryogenic fiber to between a few hundred to $1000 \mathrm{~g} / \mathrm{cm}^{3}$ at nearly Fermi degenerate conditions. Theory at the time [5] suggested that the cool, collapsed pinch might be resistively stabilized against violent MHD instability (sausage and kink modes) since the magnetic Reynolds number, the ratio of diffusion time to Alfven wave time scales, could be fairly small. While the final pinch might be stabilized, the collapse could be expected (and observed) to remain unstable so that high density could only occur in very localized regions. Only high density regions have the reactivity to be relevant to ignition and burn. At a current of about 20 Megamperes, the models indicated the compressed DT could reach $\rho R \approx 1$, sufficient for isochoric ignition if a heat source could rapidly bring the fuel to fusion temperatures. Consulting equation 1 , and inserting $\rho R=1, T=$ $5 \mathrm{keV}$ one finds an ignition spot energy of $10 \mathrm{~kJ}$ at a density of $500 \mathrm{~g} / \mathrm{cc}$ and pressure of $1900 \mathrm{GBar}$. The new short pulse laser technology seemed a good candidate as a heat source, since it could deliver the energy before the fuel could disassemble, although there were many questions about the coupling efficiency. The inward propagation of energy from the critical surface would also be complicated by the very strong magnetic field that may localize even highly relativistic electrons transverse to the field. The fiber pinch (in 1D) had the advantage of a sharp density gradient at the edge of the fuel, as shown in Figure 5. The steep profile meant the critical surface for the laser was close to the pinch surface so that the ignition energy was deposited near where it was needed in the dense fuel. The sharp gradient is caused by the resistive electric field, giving a strong inward-directed Poynting flux. Low-density matter is swept inward by the associated $E \times B$ velocity. In discussions on the subject, John Nuckolls suggested replacing the pinch with laser compression. It was not clear how to get through the extended corona in the laser-compressed case to deposit the energy in the dense fuel. Max Tabak independently invented the Nuckolls version of the idea and added the ideas of a hole boring 
laser or a focus cone to allow coupling through the corona. The different efforts were combined and Fast Ignition at Livermore was launched [6].

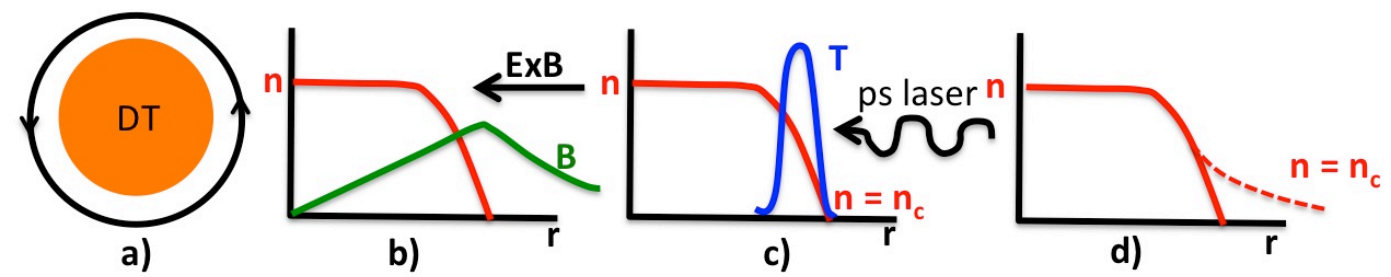

Figure 5. a) Collapsed pinch in cross-section b) Density and magnetic field profile. c) Heating to ignition with a short pulse laser d) Residual low $\beta$ instability draws out a corona

The fiber pinch version of Fast Ignition fell out of favor for several reasons. There are practical difficulties in collocating the short pulse laser pulse in space and time with the radiatively collapsed regions. The more recent development of the X-pinch might ease that difficulty. Also, further work on z-pinch stability [7] showed that, while the high density portions of the pinch might be resistively stabilized, the low density edge remains unstable. It is well known from MHD theory that at low $\beta$, where $\beta$ is the ratio of plasma to magnetic pressures, curvature or gravitational instabilities (e.g., sausage and Rayleigh Taylor modes) become electrostatic in nature. The electrostatic modes have negligible perturbed current and hence no resistive stabilization. The instability would likely pull out a plasma corona and complicate laser coupling, as sketched in figure 5(d).

Study of Fast Ignition led the author to a broader exploration of fusion applications of pulsed power. In the next section we will see how pulsed power leads us back to possibilities for indirect drive ICF.

\section{Pulsed power hohlraum-driven fusion}

It has been known for many years that imploding fast pinches couple strongly to radiation and produce some of the brightest $\mathrm{x}$-ray sources in existence [8]. The work done on the plasma by magnetic forces is mostly radiated at stagnation. As noted above the z-pinch is typically strongly unstable, yet it appears that the unstable dynamics actually enhance conversion of magnetic to kinetic energy by providing additional degrees of freedom for the conversion. The instability time scales are of order the Alfven transit time, which is comparable to stagnation times of the pinch. The inward $E \times B$ Poynting flux discussed above is enhanced by instability, drawing much of the magnetic energy of the system into the stagnation, and hence efficiently into x-rays. Experiments at Sandia National Laboratories on the Saturn and $\mathrm{Z}$ facilities showed the $\mathrm{x}$-ray sources could be used to heat hohlraums.

Of particular interest were vacuum hohlraums where the collapsing pinch drives an enclosing hohlraum, leading to the possibility that these could be a candidates for indirect drive [9]. Key parts of the concept were to place pinches in primary hohlraums, on each end of a secondary hohlraum containing the capsule, with a Faraday cage barrier between hohlraums. The Faraday cage is an array of narrow radial spokes or a metal web that is largely transparent to $\mathrm{x}$-rays but prevents magnetic fields from perturbing the secondary hohlraum and capsule. The pinches reside behind shine shields so that the capsule has no direct view of the pinch and the small-scale non-uniformities of the pinch emission are highly smoothed by the hohlraum. Symmetry is tuned through hohlraum geometry, e.g. length to diameter of the secondary hohlraum and pulse shaping is achieved by adding shells to the imploding pinch. Each of these aspects of the concept was successfully tested in a remarkable series of experiments at Sandia [10]. For example, symmetry control to $\sim 3 \%$ was demonstrated.

The difficulty with the vacuum hohlraum concept is in reaching high radiation temperatures. The design with $16 \mathrm{MJ}$ of pinch energy reached secondary temperatures of $220 \mathrm{eV}$, sufficient to give ignition and high yield at convergence ratios comparable to the nominal NIF ignition target. If it were possible to reach higher temperatures, e.g. $\sim 300 \mathrm{eV}$, then the energy advantage of the pinch could be of great value for adding margin as discussed in section 1. Figure 6 shows such a scheme, where the 
imploding pinches are shifted axially by guide cones in order to flow into primary hohlraums with small wall area, and correspondingly small wall loss, compared to the original concept. The concept is similar to one proposed by Chittenden [11].
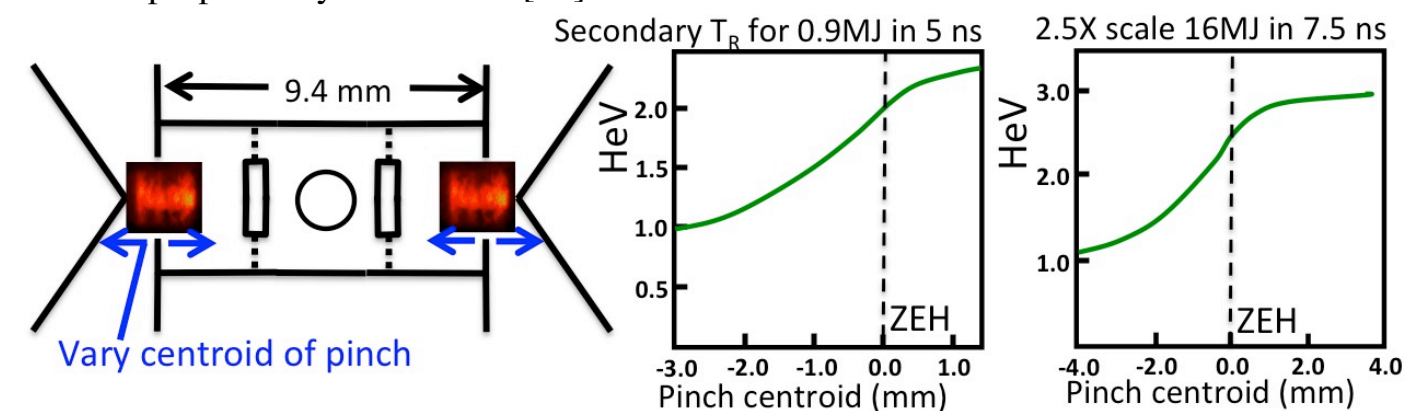

Figure 6. Double-ended vacuum hohlraum geometry where the pinch is projected axially through an entrance hole, the ZEH, into a small primary hohlraum. Middle image shows secondary temperatures versus pinch location for Z-like energy. The rightmost image shows secondary temperatures for a $2.5 \mathrm{X}$ linear scale of the $\mathrm{Z}$-scale hohlraum with $16 \mathrm{MJ}$ of pinch energy.

The radiation temperatures in Figure 6 are derived from a simple wall loss and coupled hohlraum model [11]. The capsule in the $2.5 \mathrm{X}$ scale hohlraum with $16 \mathrm{MJ}$ pinch sources absorbs nearly $3 \mathrm{MJ}$ and is driven at close to $300 \mathrm{eV}$, suggesting a potential way of reaching the robust ignition regime for the Euler scaled high foot capsule shown in Figure 1.

\section{Conclusions}

HED fusion is rich with multiple approaches, and exciting progress is occurring on the flagship NIF facility. Experimental progress points to the possible paths ahead toward ignition, as sketched in figure 2. The paths include improvements in implosion quality and hohlraum control on the NIF, but may well also include other approaches such as pulsed-power driven indirect drive.

\section{Acknowledgements}

The author thanks Omar Hurricane, Steve MacLaren, Marilyn Schneider, Paul Springer, John Lindl, Max Tabak, Mordy Rosen, Aaron Miles, Mark Herrmann, Dan Sinars and Mike Cuneo for many useful discussions. A special thanks to John Nuckolls for many years of serving as mentor and instilling "fusion by any means". This work was performed under the auspices of the U.S. Department of Energy by Lawrence Livermore National Laboratory under Contract DE-AC52-07NA27344.

\section{References}

[1] Lindl J 1998 Inertial Confinement Fusion (New York: Springer-Verlag)

[2] Cerjan C, Springer P, Sepke S 2013 Phys. Plasmas 20056319

[3] Hurricane O 2013 Bull. Am. Phys. Soc. 5816

[4] Hammel J, Scudder D 1987 Proc. $14^{\text {th }}$ Eur. Conf. Contrl. Fus. Plasma Phys., Madrid p.450

[5] Haines M, et al., 1989 Proc. $2^{\text {nd }}$ Intl. Conf. on Dense Z-pinches, Laguna Beach p.203

[6] Tabak M, Hammer J, et al., 1994 Phys. Plasmas 11626

[7] Hammer J, et al., 1996 Phys. Plasmas 32063

[8] Matzen K, et al., 2005 Phys. Plasmas 12055503

[9] Hammer J, Tabak M, et al., 1999 Phys. Plasmas 62129

[10] Cuneo M, et al., 2006 Plasma Phys. Control. Fusion 48 R1-R35

[11] Chittenden J, et al., 2002 Phys. Rev. Lett. 88235001

[12] Rosen, M 1999 Phys. Plasmas 61690 